\title{
A Humanoid Robot Companion for Wheelchair Users
}

\author{
Miguel Sarabia and Yiannis Demiris \\ Personal Robotics Lab \\ Department of Electrical and Electronic Engineering \\ Imperial College London, United Kingdom \\ \{miguel.sarabia, y.demiris\}@imperial.ac.uk
}

\begin{abstract}
In this paper we integrate a humanoid robot with a powered wheelchair with the aim of lowering the cognitive requirements needed for powered mobility. We propose two roles for this companion: pointing out obstacles and giving directions. We show that children enjoyed driving with the humanoid companion by their side during a field-trial in an uncontrolled environment. Moreover, we present the results of a driving experiment for adults where the companion acted as a driving aid and conclude that participants preferred the humanoid companion to a simulated companion. Our results suggest that people will welcome a humanoid companion for their wheelchairs.
\end{abstract}

\section{Introduction}

Driving a wheelchair is a cognitively challenging task [7]. Users need to model the behaviour of the wheelchair, predict how it is going to behave and be spatially aware of their surroundings. Could there be a way of reducing the cognitive requirements driving a wheelchair demands? We hypothesise that a humanoid robot may be able to do so. This way, we aim to lower a wheelchair's entry barrier for people with cognitive disabilities and children whose cognitive faculties may not be fully developed.

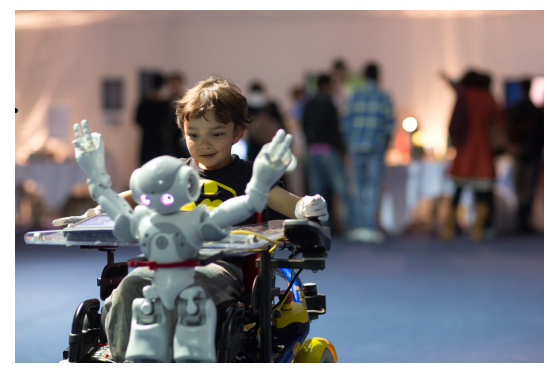

To that end we have added Nao, a small during the Imperial Festival ${ }^{2}$. humanoid, to a paediatric wheelchair so that it can act as a companion for mobility-impaired persons ${ }^{1}$. In particular, we present two possible roles for Nao. In the first one, Nao points out the location of obstacles, explaining why the smart wheelchair may not be moving in the expected direction. For the second one, we set it to act as a driving aid giving directional instructions and compare it to more traditional driving aids (such as voice and on-screen arrows).

\footnotetext{
${ }^{1}$ This work was partially funded by the EU FP7 ALIZ-E project (248116).

${ }^{2}$ Videos of the system are available at: http://imperial .ac.uk/PersonalRobotics.
} 
We are also interested in understanding whether there are any benefits to having a physical robot as a companion rather than a simulated one. Our results will show that, at least for adults, participants much preferred the physical robot over the simulated one, even if the performance differences were inconclusive.

\section{Related Work}

In 2008 , it was estimated that $61 \%$ to $91 \%$ of wheelchair users may benefit from a smart powered wheelchair at some point during their lives [12]. There is a wealth of research in the field of smart powered wheelchair reviewed in [11].

The need for powered mobility may be even more pronounced for children. Indeed, the Rehabilitation Engineering \& Assistive Technology Society of North America supports the use of paediatric powered mobility as soon as the child possesses the necessary cognitive, sensorimotor and coping abilities [10]. According to the same study, the use of smart powered wheelchairs "enhances independence, improves psychosocial development and enables children to become productive and independent members of society".

It has been shown that a robotic wheelchair with haptic guidance can be used to teach children to drive [5]. This system, based on a game of "robot-tag", was used by 22 able-bodied children and a 8 year old with cerebral palsy. ARTY (Assistive Robotic Transport for Youngsters) is a paediatric wheelchair with a focus on safety. It was tested by 8 able-bodied children and a 5 year old with physical and cognitive disabilities [13]. Note both wheelchairs were transferred from able-bodied to cognitively impaired children. Further evidence of the potential of smart wheelchairs comes from [8] where 4 children with cerebral palsy aged 11 to 16 successfully navigated around their school using a touch-screen.

To the best of our knowledge there is little published research in companions for robotic wheelchair users. Thus, we review two European Framework Programme projects on robotic companions. IROMEC (Interactive Robotic Social Mediators as Companions) showcases the benefits of a robotic companion able to play with developmentally-impaired children [6]. CompanionAble, on the other hand, seeks to assist people in their homes and it is targeted for the elderly, particularly those who suffer from mild cognitive impairment [4].

Additionally, in 2007 the car manufacturer Nissan, added a PaPeRo robot to their Pivo 2 concept car to provide driving assistance. Whilst this was well reported on the press, we were unable to find any relevant scholarly material.

Finally, there has been research into the differences between a simulated robot and a physical one. In particular, it has been shown that people rate interactions

with an actual robot more positively than with one seen through a live video display [1].

\section{System Description}

Our system has two distinct subsystems: ARTY and Aldebaran's Nao (Fig. 2). ARTY is composed of an Ottobock children's powered wheelchair, three laser 


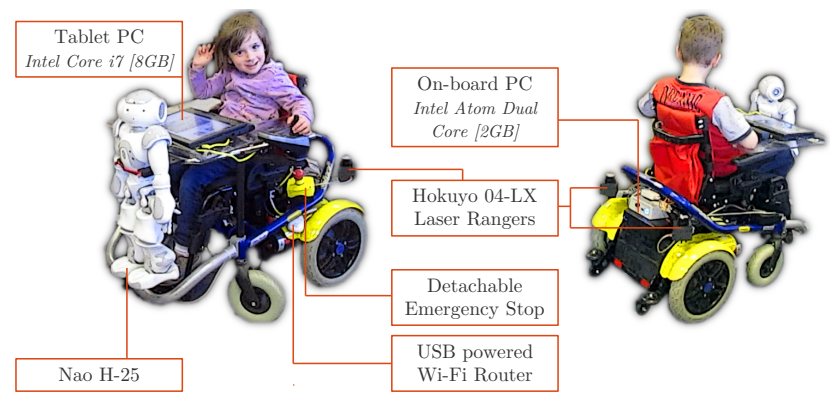

Fig. 2. Hardware components of our system.

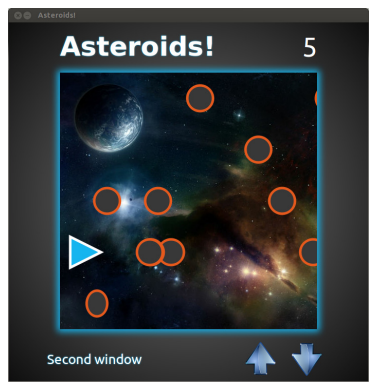

Fig. 3. Asteroids game.

rangers, an on-board PC, a tablet PC and a wireless router. ARTY is an improved version of the one presented in [13]. Nao is a $60 \mathrm{~cm}$ tall humanoid robot with 25 degrees of freedom which hangs from the front of the wheelchair.

Figure 4 summarises the main software components of our system, all of which are written atop ROS (the Robot Operating System) [9]. We reuse many software components readily available online and in what follows we describe the most relevant ones. Laser Combiner ${ }^{3}$ takes the readings of the three laser rangers and combines them into a single coherent message [13]. Laser Scan Matcher ${ }^{4}$ takes in this combined message and interpolates the wheelchair odometry using an iterative closest point algorithm [3]. AMCL ${ }^{5}$

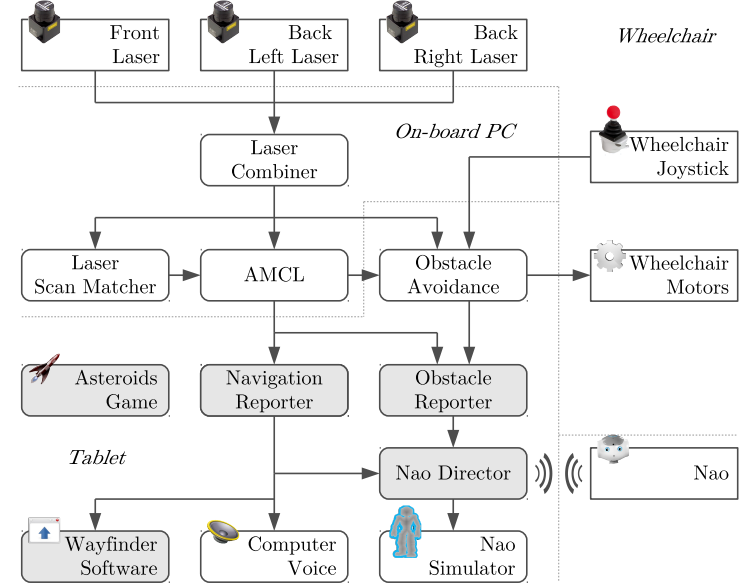

Fig. 4. Software components of our system. Square edges represent hardware, round edges represent software nodes. Shaded nodes are contributions of this article. See text for authorship of other nodes. receives both the interpolated odometry and the combined laser message to localise the wheelchair on a pre-existing map. The Obstacle Avoidance ${ }^{2}$ module moderates the input joystick signal according to the wheelchair proximity to obstacles using a dynamic window approach [13], thus avoiding potential collisions.

\footnotetext{
${ }^{3}$ Nodes written by Harold Soh, available from http://imperial.ac.uk/PersonalRobotics.

${ }^{4}$ Node written by Ivan Dryanovski and William Morris, available from http: //www.ros.org/wiki/laser_scan_matcher.

${ }^{5}$ Node written by Brian Gerkey and Andrew Howards, available from http://www.ros.org/wiki/amcl.
} 
Let us now introduce the nodes written specifically for our system. Navigation Reporter raises an alert whenever the user has deviated from a pre-recorded path on the map and indicates the direction the user should follow at the junctions of said path. Every time a new pose is generated by the AMCL node, Navigation Reporter tries to find its closest match in the list of poses that compose the predefined path. To achieve this, the program evaluates all path poses within \pm 1.5 metres of the last known path pose and chooses the one with highest score. The scoring function is defined as: score $=\exp \{\Delta / \alpha \times \ln 2\}+\exp \{\Gamma / \beta \times \ln 2\}$ where $\Delta$ represents the euclidean distance between the current wheelchair pose and the candidate path pose, $\Gamma$ is the normalised angle difference between the current wheelchair pose and the candidate path pose; $\alpha$ and $\beta$ are adjusting variables to control what distance and angles difference yield half score (we set $\alpha=0.4 \mathrm{~m}$ and $\beta=\pi / 4 \mathrm{rad}$ respectively, these values were determined empirically). If the score of all path poses considered was lower than a threshold (set to 1.3) no pose was chosen. This formulation is robust to errors in AMCL and can deal with paths that go over the same point repeatedly (thanks to the $\pm 1.5 \mathrm{~m}$ sliding window).

Obstacle Reporter simply takes the information from the Obstacle Avoidance node and raises an alert if the user is driving towards an obstacle. To prevent Nao from becoming too repetitive, both Obstacle Reporter and Navigation Reporter suppress similar alerts that occur in a short period of time.

The asteroids game (Fig. 3) was developed as a secondary task for the path driving experiments (introduced in Sect. 5). The objective is to move the spaceship (triangle in the figure) away from the incoming asteroids (circles in the figure) using the up and down arrows.

\subsection{Driving Aids: Pointing out Obstacles and Giving Directions}

Here we introduce the nodes in charge of communicating wheelchair alerts to the user. We start with Nao Director, which coordinates all of Nao's movements and speech. It instructs Nao to execute wake-up and power-off animations and provides a background behaviour for Nao - randomly looking around and blinking. If the node receives any alert from either Navigation Reporter or Obstacle Reporter it will stop the background behaviour and command Nao to indicate the direction the user has to follow (in case of a navigation alert) or the location of the obstacle (in case of an obstacle alert).

Nao Director can command both the actual Nao or an on-screen simulated Nao. The underlying code is the same. However the simulated Nao cannot emit sounds and instead writes them on the display. To minimise the differences between the simulated Nao and the physical one, Nao Director did not provide new information through voice, instead giving vague messages (eg. "we have to go that way"). One has to look at the arms of the robot or the simulator in order to understand its instructions.

We also implemented two other, more traditional, driving aids. The first one is the Wayfinder Software, a simple computer window to show navigation alerts. Upon receiving an alert, an arrow indicating the direction the user should follow is shown on-screen for 3 seconds. The second driving aid was the Computer 
Voice, a program which would speak out loud the instructions received from Navigation Reporter using ROS text-to-speech facilities. Example utterances are: "go straight" and "drive left". The sound of the ROS computer voice was easily distinguishable from that of Nao.

\section{Preliminary Field Trials}

During our university's open-day in May 2013, we conducted trials of our wheelchair plus companion system with several able-bodied children. Since we had very little control over the environment (it was estimated that 10,000 people attended the event) the main focus of the exercise was to check whether children appreciated driving with $\mathrm{Nao}$ as a companion. Note Navigation Reporter was disabled and Nao only reported the location of obstacles.

We asked 14 participants, who had driven the wheelchair for about 5 minutes each, to fill in a questionnaire with three questions in five-point Likert-scale ${ }^{6}$. The responses came from 9 boys and 5 girls aged 4 to 12. Some of the younger participants were helped by their parents when filling in the questionnaire. Children were generally very positive about the field-trials: all 14 children strongly agreed they had enjoyed the wheelchair, 12 strongly agreed Nao had helped them not to crash and 11 participants strongly agreed they liked having Nao by their side when driving. The participants also had the opportunity to write comments about the experiment. All comments were positive; for example: "I want him as a pet or brother", "Nao is really helpful", "I think HE IS AWESOME!".

Although there was an inherent pressure on the children to evaluate the wheelchair positively (it is hard to criticise the toy one has just played with), we consider the enthusiastic comments to be a proof that, at least in the short term, children really enjoy having Nao as a companion.

\section{$5 \quad$ Path Driving Experiments}

In this section we explore the effects of having a humanoid companion as a driving aid. We also investigate whether there was any advantage to having a physical robot rather than a simulator.

\subsection{Experimental Set-up}

Participants were tasked with navigating through a pre-defined path. The basic make-up of each path was the same, all shared the starting point and driving segments, the difference came from

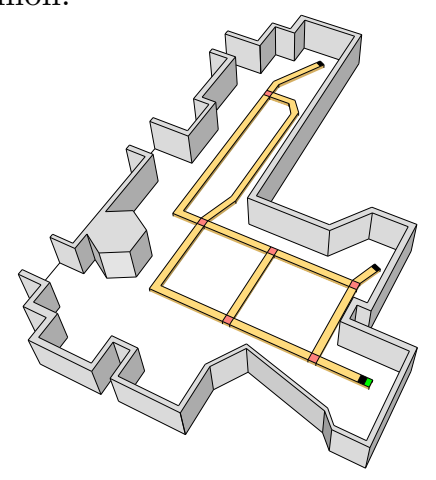

Fig. 5. Path-driving environment with 6 junctions and 3 finishing points (in black). the turns to be performed at each junction and the finishing point. There were

\footnotetext{
${ }^{6}$ To make the questionnaire friendlier we used smileys instead of the typical 'strongly disagree' to 'strongly agree' scale.
} 
a total of 6 junctions and 3 finishing points (Fig. 5). All paths were designed so it would take around three minutes to complete them.

The participants did not know the exact directions to follow on the course, instead relying on different driving aids to guide them through the pre-defined paths. Specifically, driving aids told users to turn left, right or keep going straight at each junction. If participants did not follow the instructions the system would remember the last point where they had been on-course and instructed them to retrace their steps until they reached a known point (lost instruction).

To simulate real wheelchair driving conditions where a user may be distracted by some other task (eg. by having a conversation) and following the example set in [2], we devised a secondary task: the asteroids game introduced in Sect. 3.

Participants were asked to complete four paths. Each time, they were aided by one of the driving aids already described in Sect. $3.1^{7}$. At the end of each trial, they were asked to complete a questionnaire regarding the driving aid they had just used. Additionally, at the end of the experiment they were requested to chose their preferred driving aid.

Throughout these experiments Obstacle Reporter was disabled and Nao only acted as a driving aid. This was done as a control measure since neither of the traditional driving aids could communicate obstacle alerts. All data interchanged by the different ROS nodes of the system, including scores on the asteroids game, was recorded for subsequent analysis of user performance.

\subsection{Performance Metrics and Questionnaires}

The following metrics are used to compare the different driving aids:

Average driving speed computed as the sum of the euclidean distances between the poses reported by ACML over the time the trial lasted. A higher value indicates better overall performance in the task.

Impacts defined as number of impacts in the secondary task. A high score indicates participants attention was occupied with driving. Accordingly, a lower value implicates better overall performance.

Time lost computed as sum of the durations between a user receiving a lost instruction and the user receiving any other instruction, which only happens when the participant has driven back to the junction where she became lost. A lower value is suggestive of better overall performance.

We also collected subjective metrics through the use of questionnaires. The questions are listed below (note system was replaced by the actual driving aid):

- "I found driving the wheelchair whilst playing the game difficult".

- "I felt safe in the wheelchair".

- "I understood the instructions the system was trying to convey".

- "The system gave me accurate instructions".

\footnotetext{
${ }^{7}$ The order each participant used every driving aid was determined by choosing a random entry in a table with all permutations of driving aids.
} 


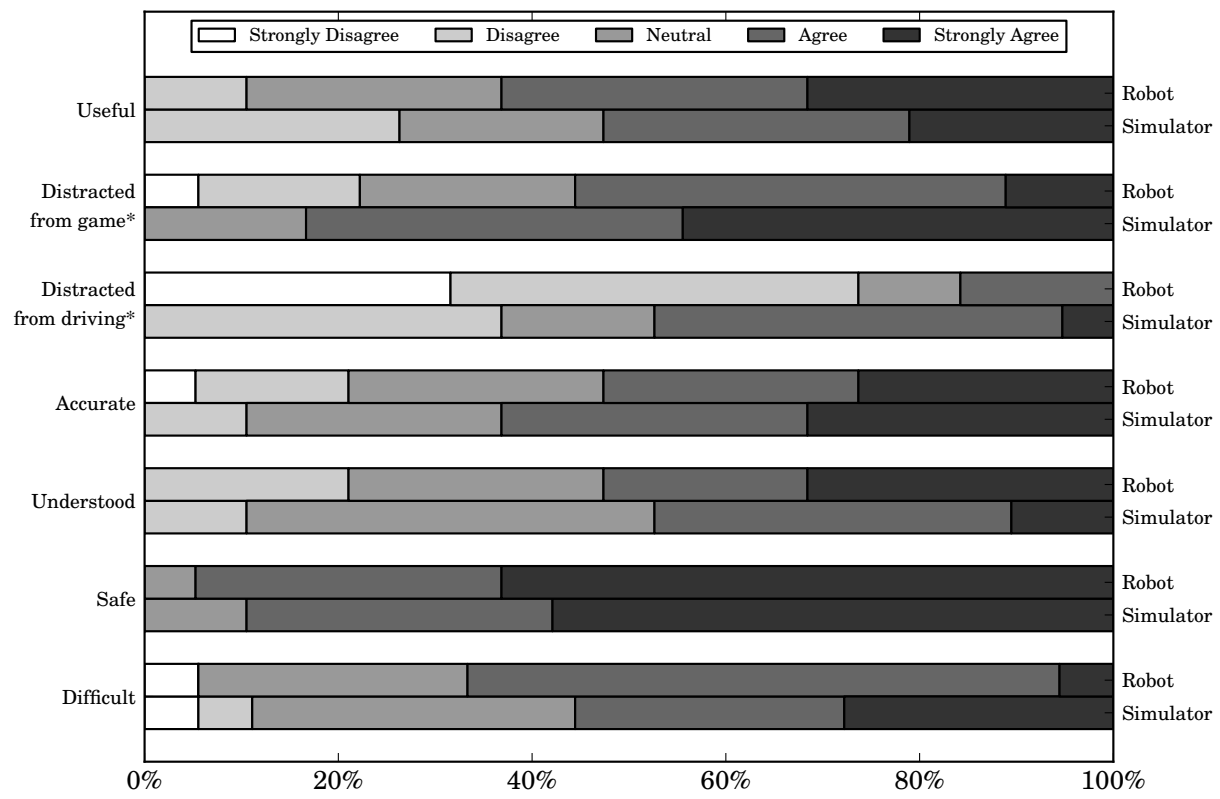

Fig. 6. Questionnaire responses for Simulator and Nao considering all attempts. Categories appended with * are statistically significant. See Sect. 5.2 for actual questions.

- "The system distracted me from driving".

- "The system distracted me from playing the game".

- "I found the system to be a useful driving aid".

\subsection{Results}

20 people (11 female and 9 male) aged between 21 and 38 completed the experiment. $40 \%$ of the participants declared to have worked with robots before. Due to a misaligned laser scanner we had to discard one of the trials as the AMCL module failed to localise properly.

Even though participants had a few minutes practice with the wheelchair before the actual experiment started, we found most of the learning occurred during the first attempt. This is evident from Fig. 7 where the deviation from the average performance is greatest in the first attempt for all three metrics. Therefore, we report on data distilled from all attempts as well as from the last three attempts.

Figure 6 shows the questionnaire results considering all attempts $(N=19)$. Safety and accuracy have equivalent scores for both the simulator and the robot. When asked about difficulty, understanding or usefulness, ratings are generally higher for the Nao robot but the differences do not reach statistical significance ${ }^{8}$. In contrast, the robot has significantly lower ratings for distracting from driving $(p=0.007, W=11)$ and distracting from game $(p=0.032, W=14.5)$.

\footnotetext{
${ }^{8}$ We used the Wilcoxon signed-rank matched pairs test which is a non-parametric, within-subjects and two-tailed test.
} 


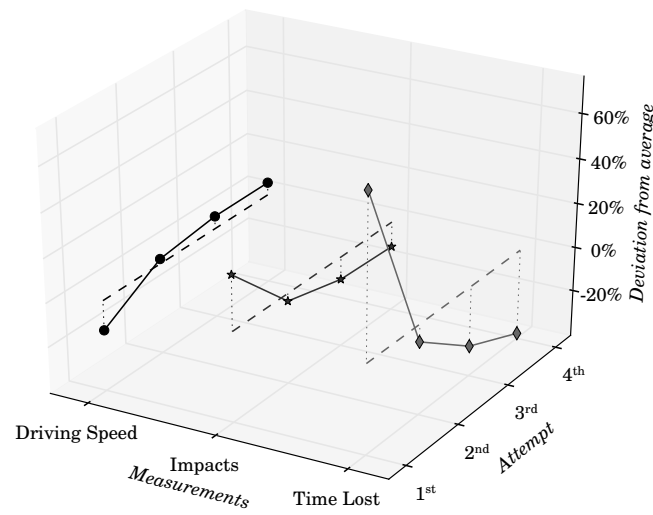

Fig. 7. Deviation from average across all trials in driving performance. Results from different driving aids have been aggregated.

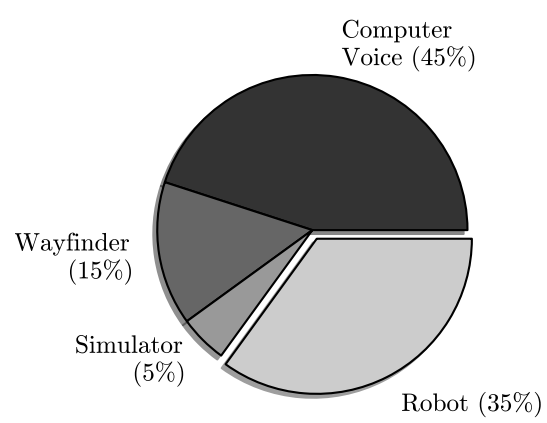

Fig. 8. Preferred driving aids.

It is noteworthy that when considering only the last three attempts $(N=9)^{9}$ both distracted from driving and distracted from game were no longer statistically significant, though they still favoured the robot Difficulty and understanding continued to have higher, non-significant, ratings for the robot, whereas safety and accuracy had very similar ratings. Importantly, $89 \%$ agreed or strongly agreed that the robot was useful compared to only $33 \%$ for the simulator; this was found to be statistically significant $(p=0.031, W=21)$.

With respect to the data recorded, we will indicate the median and the interquartile ranges (in square brackets) for driving speed $(v)$, impacts $(i)$ and time lost $(\tau)$. Note do not assume normality. Our data shows that when considering all trials $(N=19)$ the median driving speed was $\tilde{v}_{\text {robot }}=0.19 \mathrm{~m} / \mathrm{s}[0.10 \mathrm{~m} / \mathrm{s}]$ and $\tilde{v}_{\text {sim }}=0.21 \mathrm{~m} / \mathrm{s}[0.11 \mathrm{~m} / \mathrm{s}]$ for the Nao robot and the Nao simulator. Similarly, the median number of impacts was $\tilde{i}_{\text {robot }}=69$ [45.75] and $\tilde{i}_{\text {sim }}=64$ [60.5]; the time lost was $\tilde{\tau}_{\text {robot }}=21.91 \mathrm{~s}[82.69 \mathrm{~s}], \tilde{\tau}_{\text {sim }}=8.27 \mathrm{~s}[72.53 \mathrm{~s}]$. If only the last three trials are considered $(N=9)$ we find that $\tilde{v}_{\text {robot }}=0.24 \mathrm{~m} / \mathrm{s}[0.08 \mathrm{~m} / \mathrm{s}]$, $\tilde{v}_{\text {sim }}=0.22 \mathrm{~m} / \mathrm{s}[0.07 \mathrm{~m} / \mathrm{s}] ; \tilde{i}_{\text {robot }}=52[55], \tilde{i}_{\text {sim }}=56[43] ; \tilde{\tau}_{\text {robot }}=0 \mathrm{~s}[15.86 \mathrm{~s}]$, $\tilde{\tau}_{\text {sim }}=30.73 \mathrm{~s}[57.49 \mathrm{~s}]$. None of these differences were found to be statistically significant.

We were also interested in whether either the Nao robot or the Nao simulator would yield better performance than the control driving aids. Our data shows that the wayfinder software had the best median performance metrics $\left(\tilde{v}_{\text {wayfinder }}=0.24 \mathrm{~m} / \mathrm{s}[0.06 \mathrm{~m} / \mathrm{s}], \tilde{i}_{\text {wayfinder }}=49[43.25], \tilde{\tau}_{\text {wayfinder }}=5.57 \mathrm{~s}[30.64 \mathrm{~s}]\right)$, followed by the computer voice $\left(\tilde{v}_{\text {voice }}=0.22 \mathrm{~m} / \mathrm{s}[0.08 \mathrm{~m} / \mathrm{s}], \tilde{i}_{\text {voice }}=52\right.$ [43.25], $\tilde{\tau}_{\text {voice }}=7.23 \mathrm{~s}[23.93 \mathrm{~s}]$ ), followed by the Nao simulator and Nao robot (see above for medians and interquartile ranges). Performing Friedman tests reveals a statistically significant effect of the driving aid on all three performance metrics. We

\footnotetext{
${ }^{9}$ This corresponds to the number of people who did not have either the Nao robot or the Nao simulator on the first attempt.
} 
found $T=10.45$ and $p=0.015$ for driving speed; $T=12.97$ and $p=0.005$ for impacts; and $T=9.41$ and $p=0.024$ for time lost. Notice that, again, $N=19$.

Figure 8 shows the preferred driving aids of participants. Remarkably, the robot was $30 \%$ ahead of the Nao simulator.

\subsection{Discussion}

The robot and the simulator had very similar scores in safety and accuracy. This was expected as safety depends on the Obstacle Avoidance module which was active throughout the experiment. Likewise, the accuracy of instructions relies upon the Navigation Reporter which is shared by both simulator and robot.

In most other categories the robot had a slight advantage in ratings when compared to the simulator, though most of these differences did not reach statistical significance. The exceptions were distraction and usefulness.

Interestingly, the robot scored significantly lower in the distracted from game category than the simulator. This is surprising since the simulator shared the screen with the game and participants did not have to look away. Moreover, even if the accuracy and the actual task performance was similar across both driving aids, users much preferred the robot over the simulator (30\% difference when it came to the favourite driving aid). Might the lack of embodiment cause the simulator to be more distracting and less useful? Further research is needed to clarify this question.

Although the simulator presented higher driving speed, lower number of impacts and less time lost, none of the results were statistically significant. Moreover when considering the last three attempts the robot had a slightly higher driving speed and a considerably lower time lost, suggesting more time may be required to habituate to Nao.

Taking into account the traditional driving aids it is clear that the robot and the simulator were not as effective as the control driving aids. More work is needed to ensure these robotic driving aids catch up with or even improve upon their traditional counterparts.

To be specific, we identified two issues which might explain why the performance of Nao was lower than that of the control driving aids. Firstly, Nao sometimes took too long to give instructions due to unpredictable network latency, which confused participants. Secondly, many participants were disappointed and mentioned in the comments that Nao never gave instructions by voice. By exploiting multi-modality Nao may become a more effective driving aid.

\section{Conclusion and Further Work}

We have investigated the potential of a humanoid companion for wheelchairs. Children were highly positive about having a robot accompanying them during an uncontrolled field trial. A physical robot was also found by users to be more useful and less distracting than a simulated one. This was the case even when there was no statistically significant indication of performance improvements. 
In the future, we look forward to formulating new companion roles (besides the roles of obstacle reporter and driving aid we presented here) where embodiment is not only advantageous but a requirement. Having shown that able-bodied users appreciate a humanoid companion, we will be testing to verify how well our findings carry over to disabled children and people with cognitive disabilities. This is an area where robotic companions have a particular potential to greatly improve the quality of life of wheelchair users as well as lowering the entry barrier to powered mobility, allowing people that currently cannot drive a wheelchair to do so.

\section{References}

1. Bainbridge, W.A., Hart, J.W., Kim, E.S., Scassellati, B.: The Benefits of Interactions with Physically Present Robots over Video-Displayed Agents. Intl J Social Robot. 3(1), 41-52 (2010)

2. Carlson, T., Demiris, Y.: Collaborative control for a robotic wheelchair: evaluation of performance, attention, and workload. IEEE Trans. Syst., Man, Cybern., Part B 42(3), 876-888 (2012)

3. Censi, A.: An ICP variant using a point-to-line metric. In: Proc of IEEE ICRA. pp. 19-25 (2008)

4. Gross, H.M., Schroeter, C., Mueller, S., Volkhardt, M., Einhorn, E., Bley, A., Langner, T., Martin, C., Merten, M.: I'll keep an eye on you: Home robot companion for elderly people with cognitive impairment. In: Proc of IEEE SMC. pp. 2481-2488 (2011)

5. Marchal-Crespo, L., Furumasu, J., Reinkensmeyer, D.J.: A robotic wheelchair trainer: design overview and a feasibility study. J Neuroeng. Rehabil. 7(40) (2010)

6. Marti, P., Giusti, L.: A Robot Companion for Inclusive Games: a user-centred design perspective. In: Proc of IEEE ICRA. pp. 4348-4353 (2010)

7. Massengale, S., Folden, D., McConnell, P., Stratton, L., Whitehead, V.: Effect of visual perception, visual function, cognition, and personality on power wheelchair use in adults. Assist. Technol. 17(2), 108-21 (2005)

8. Montesano, L., Díaz, M., Bhaskar, S., Minguez, J.: Towards an intelligent wheelchair system for users with cerebral palsy. IEEE Trans. on Neural Syst. Rehabil. Eng. 18(2), 193-202 (2010)

9. Quigley, M., Gerkey, B.P., Conley, K., Faust, J., Foote, T., Leibs, J., Berger, E., Wheeler, R., Ng, A.: ROS: an open-source Robot Operating System. In: Proc Open Source Software Workshop at ICRA (2009)

10. Rosen, L., Arva, J., Furumasu, J., Harris, M., Lange, M.L., McCarthy, E., Kermoian, R., Pinkerton, H., Plummer, T., Roos, J., Sabet, A., Vander Schaaf, P., Wonsettler, T.: RESNA position on the application of power wheelchairs for pediatric users. Assist. Technol. 21(4), 218-225 (2009)

11. Simpson, R.C.: Smart wheelchairs: A literature review. J Rehabil. Res. Dev. 42(4), 423-438 (2005)

12. Simpson, R.C., LoPresti, E.F., Cooper, R.A.: How many people would benefit from a smart wheelchair? J Rehabil. Res. Dev. 45(1), 53-72 (2008)

13. Soh, H., Demiris, Y.: Towards Early Mobility Independence: An Intelligent Paediatric Wheelchair with Case Studies. In: Proc IROS Workshop on Progress, Challenges and Future Perspectives in Navigation and Manipulation Assistance for Robot Wheelchairs. Vilamoura, Portugal (2012) 